\title{
Calidad y demanda del servicio de hoteles de tres estrellas en la región petrolera de Ciudad del Carmen, Campeche
}

\section{Quality and Demand for the Service of Three-star Hotels in the Oil Region of Ciudad del Carmen, Campeche}

\author{
David Martínez Luis* (1) https://orcid.org/0000-0002-4747-9368 \\ Alberto Pérez Fernández ${ }^{* *}$ (D) https://orcid.org/0000-0002-9397-4167 \\ Ignacio Caamal Cauich $^{* * *}$ (i) https://orcid.org/0000-0002-3571-0542
}

\begin{abstract}
Resumen
El objetivo de la investigación es evaluar la calidad del servicio y los factores que afectan la demanda de hoteles de tres estrellas en Ciudad del Carmen, Campeche. La calidad del servicio se evaluó a partir del modelo Servqual y la demanda se analizó utilizando un modelo de ecuaciones simultáneas. Los resultados muestran la dificultad de los hoteles para satisfacer a los huéspedes y que la calidad depende de los aspectos físicos del hotel y del trato personal. La elasticidad precio de la demanda es inelástica y el ingreso (remuneración) de los huéspedes tiene una relación inversa con la demanda de habitaciones. La investigación tiene como limitante que sólo estudia el mercado de hoteles desde un enfoque de demanda, pero aporta elementos para redefinir estrategias en empresas de servicios turísticos. Se concluye que un mercado seguro no siempre incentiva la mejora de la calidad; en algunos casos puede ser una limitante para la diversificación económica de una región.
\end{abstract}

Palabras clave: hoteles; Servqual; calidad del servicio; elasticidad; precio; ingreso.
Abstract

The objective of this research is to evaluate the service quality and the factors that affect the demand for three-star hotels in Ciudad del Carmen, Campeche. Servqual model was used to evaluate service quality and a simultaneous equation model helped during the demand analysis. Results show that hotels have difficulty to satisfy guests' needs. Demand depends on the hotel's physical aspects and on the treatment personnel gives to lodgers. The demand's price flexibility is inelastic and income has an inverse relationship with the demand for rooms. The research limitation is that it only studies the hotel market from a demand perspective, but provides elements to redefine strategies in tourist service companies. It is concluded that a secure market does not always incentivize quality improvement; in some cases, it can be limiting for the economic diversification of a region.

Keywords: hotels; Servqual; service quality; elasticity; price; income.

Cómo citar: Martínez Luis, D., Pérez Fernández, A., y Caamal Cauich, I. (2020). Calidad y demanda del servicio de hoteles de tres estrellas en la región petrolera de Ciudad del Carmen, Campeche. región y sociedad, 32, el 259. doi: I0.22 I98/rys2020/32/I259

* Autor para correspondencia. Universidad Autónoma del Carmen, Facultad de Ciencias Económicas Administrativas. Calle 56, Núm. 4, Esq. Avenida Concordia, Col. Benito Juárez, C. P. 24180, Ciudad del Carmen, Campeche, México. Correo electrónico: dmartinez@pampano.unacar.mx

** Universidad Autónoma del Carmen, Facultad de Ciencias Económicas Administrativas. Calle 56, Núm. 4. Esq. Avenida Concordia, Col._Benito Juárez, C. P. 24180. Ciudad del Carmen, Campeche, México. Correo electrónico: apfernandez@pampano.unacar.mx

*** Universidad Autónoma Chapingo, División de Ciencias Económico-Administrativas. Carretera México-Texcoco, Km. 38.5, C. P. 56230, Texcoco, Estado de México, México. Correo electrónico: icaamal82@yahoo.com.mx 


\section{Introducción}

La industria hotelera ha tenido un fuerte dinamismo en México. De acuerdo con la Secretaría de Turismo (SECTUR, 2018), entre 1995 y 2018 los establecimientos de hospedaje crecieron $155.8 \%$ y el número de cuartos disponibles aumentó $118.2 \%$. El crecimiento de establecimientos de tres estrellas fue de $226.1 \%$, la cantidad de cuartos disponibles incrementó $131.6 \%$ y el porcentaje de ocupación pasó de 42.4 a 48.3. La Confederación de Cámaras Nacionales de Comercio, Servicios y Turismo (CONCANACO-SERVYTUR, 2016) estimó que el sector turístico contribuyó con $9.1 \%$ de los empleos creados en el país y generó una derrama económica de 2 billones 101 mil millones de pesos en 2014. Los servicios de alojamiento temporal y de preparación de bebidas aportaron $2.3 \%$ del producto interno bruto (PIB) en 2015 (Instituto Nacional de Estadística y Geografía [INEGI], 2017). En el ámbito del turismo, los negocios tienen un fuerte crecimiento en el mundo y un enorme potencial de desarrollo (Flamarich y Duro, 2011).

Ciudad del Carmen pertenece al municipio de Carmen, uno de los cinco municipios más importantes del país por su aportación al PIB (INEGI, 2014). De acuerdo con Petróleos Mexicanos (PEMEX), el municipio aportó 54.2\% de la producción de crudo en México y 23\% del gas natural en 2011 (PEMEX, 2011). En 2017 generó 34.6\% de los ingresos petroleros para la federación (PEMEX, 2018). La importancia que tiene en el desarrollo económico del estado y de la región se traduce en una fuerte actividad económica, puesto que existen muchas empresas relacionadas con el sector petrolero que generan una gran cantidad de empleos. Por lo anterior, la ciudad tiene una alta demanda de servicios de alojamiento, alimentación y trasporte. En el estado existían 239 hoteles en 2011, de los cuales 108 se ubicaban en el municipio de Carmen con un total de 3267 cuartos (INEGI, 2012). El municipio contaba en 2012 con 25 hoteles de tres estrellas (INEGI, 2012).

Los hoteles de tres estrellas reciben a los trabajadores que se trasladan de sus lugares de origen a la ciudad y que están en espera de subir a las plataformas petroleras. Se hospedan también aquellos que provienen de las plataformas y que aguardan el momento de trasladarse a sus lugares de origen. Algunos de estos trabajadores utilizan sus propios recursos para pagar el hotel; otros reciben financiamiento de las compañías a las que prestan sus servicios. La actividad petrolera llevó a los hoteles de la ciudad a tener una ocupación mayor a 75\% (Blancas, 2016), muy por arriba del promedio nacional.

En 2014 y 2015 los precios de la mezcla mexicana de petróleo se ubicaron por debajo de los 50 dólares por barril, por lo cual disminuyeron los ingresos y la posibilidad de hacer nuevas inversiones en el sector petrolero (PEMEX, 2016). Además, en 2015 hubo un despido masivo de trabajadores en Ciudad del Carmen, debido a la reforma energética y al saneamiento financiero de PEMEX (Pérez, Rivas, Martínez y Venegas, 2018). Aunado a lo anterior, la producción de petróleo crudo disminuyó en 50.9\% entre 2013 y 2016 (PEMEX, 2016). El despido de empleados y la reducción de contratos asignados a otras empresas provocaron un efecto dominó que afectó a los hoteles, a la alimentación y el trasporte 
(Pérez et al., 2018). En el sector hotelero, la ocupación disminuyó de manera drástica a menos de 10\% (Blancas, 2016).

Los pequeños hoteles son importantes para el desarrollo económico de la ciudad. En un entorno de contracción económica, es necesario que busquen alternativas para mantenerse en el mercado y lograr un crecimiento (Freyle, 2010). Es de gran importancia evaluar la percepción de los huéspedes sobre la calidad de los servicios para mejorar la atención y atraer más clientes en un ambiente competitivo (Morillo, 2007; Ramírez, García y Cano, 2013).

Los objetivos del presente estudio son evaluar la calidad del servicio que reciben los huéspedes y analizar los factores que afectan la demanda de los hoteles de tres estrellas en Ciudad del Carmen, Campeche. La calidad de los servicios que ofrecen se determina mediante el modelo Servqual, el cual evalúa la expectativa y la percepción que tiene el huésped sobre los servicios. Los factores de la demanda se analizan a través de un sistema de ecuaciones simultáneas, en donde la cantidad de visitas a los hoteles está determinada por el precio y el ingreso o sueldo de los huéspedes.

\section{Turismo de negocios}

El turismo de negocios comprende el conjunto de corrientes turísticas cuyo motivo de viaje está vinculado con las actividades profesionales y laborales (Sebastián, Claudio y Larios, 2017). Vidal (2004) define el turismo de negocios como aquel que contempla viajes efectuados por ejecutivos, comerciantes y funcionarios con algún incentivo laboral o económico. Por su parte, Villena (2014) menciona que el turismo de negocios es un turismo profesional que implica el traslado a una ciudad diferente de la de residencia. De acuerdo con la Organización Mundial de Turismo (2008), un visitante de negocios es una persona que viaja a un destino principal distinto al de su entorno habitual, por una duración inferior a un año, con motivos de negocios y profesionales. El turismo de negocios se debe a la presencia de actividad industrial, a la existencia de importantes recursos turísticos o a eventos importantes (Marques y Santos, 2017).

El turismo de negocios incluye actividades de esparcimiento (González, 2011). Al viajero de negocios se le puede considerar un seudoturista debido a que el disfrute del lugar y del paisaje es una actividad adicional al desarrollo de su trabajo (Martín, 2011). Este tipo de turismo exige una infraestructura disponible todo el año, puesto que no obedece a temporadas que regulen la estacionalidad de la demanda turística (Morillo, 2011).

La ciudad de Monterrey es el principal centro industrial de México. Por eso registra una alta fluctuación de empresarios que van a hacer negocios (Hinojosa y Martínez, 2018). Celaya, en la región del Bajío, presenta un aumento del turismo de negocios debido al crecimiento de la industria automotriz (Castillo, López y Sánchez, 2019). En la Ciudad de México se llevan a cabo eventos que unen empresas nacionales e internacionales en centros de convenciones para hacer negociaciones o presentar innovaciones, lo cual ha generado una reorientación de actividades en torno al turismo (Salas y López, 2019). 
El turismo de negocios es uno de los más rentables y dinámicos del sector. Incluye las actividades de esparcimiento (González, 2011). Dicho turismo tiene la característica de que el consumidor no escoge el destino; por lo tanto, sus expectativas en torno a los servicios son diferentes a las de los turistas que eligen un lugar por deseo propio.

El desarrollo de proyectos de inversión que fomentan el turismo de negocios y de ocio permiten incrementar los empleos y los ingresos de la población (Mayo, Guzmán y Luna, 2016), pero es crucial que el sector hotelero se adapte a las exigencias del huésped (Šerić y Gil, 2011). Los negocios deben tener una adecuada gestión del capital humano para dar respuesta ágil a las demandas del mercado (Ruizalba, Vallespín y Pérez-Aranda, 2015). El crecimiento del número de hoteles en México (SECTUR, 2018) convierte a este sector en un mercado más competitivo. La innovación se ha constituido en la piedra angular de las empresas que aspiran a mejorar su competitividad (Ramírez, García y Cano, 2013). Por lo tanto, la satisfacción del cliente es fundamental en la competencia que enfrentan estos servicios.

Al diseñar productos turísticos rentables basados en la satisfacción de los consumidores, el turismo se convierte en un sector dinamizador de la economía y genera muchos empleos (López-Rodríguez, 2009). El aumento de la actividad turística en una región obliga a ofrecer servicios de calidad.

Calidad en los servicios de hospedaje

Los consumidores poseen dos tipos de expectativas sobre un servicio: el nivel deseado y el nivel percibido. La medición de la calidad es primordial. A veces ésta constituye sólo una práctica de supervisión de productos terminados y no se concibe como una identidad organizacional que genere empatía con el cliente (Robles, Martínez, Alviter y Ortega, 2016). El desarrollo de modelos y técnicas asociados a la medición de la calidad del servicio ha contribuido a la gestión de la calidad. Entre los modelos más importantes se encuentran el modelo nórdico (Grönroos, 1994) y el modelo Servqual (Parasuraman, Zeithaml y Berry, 1985), que han permitido obtener un carácter global de la percepción que tienen los clientes del sector hotelero. Es importante incorporar en los procesos de medición no sólo los factores del funcionamiento interno del establecimiento, sino también los factores externos que, sin pertenecer al sector hotelero en sí, intervienen en la conformación de la percepción global.

La medición de la calidad del servicio de hospedaje debe considerar la utilidad o el grado de satisfacción que obtiene el consumidor (Macdonald, Wilson, Martínez y Tossi, 2011). Mediante modelos de evaluación de servicios se pueden implementar medidas correctivas y preventivas en la empresa (Grzinic, 2007). Monfort, Defante, Oliveira y Mantovani (2013) encontraron que el análisis del perfil de los consumidores por medio de la matriz de atributos, permite determinar el nivel de satisfacción, las variables y dimensiones que deben recibir más atención y las inversiones que deben hacerse. En particular, se debe poner atención en la experiencia laboral acumulada por la plantilla en la empresa (Marchante y Ortega, 2010). Los hoteles deben desarrollar aptitudes en el per- 
sonal que tiene contacto directo con los consumidores para que, mediante el buen trato al huésped, puedan generar una oportunidad valiosa para la expansión de la red hotelera (Monfort et al., 2013).

Existen factores sobre los cuales el establecimiento puede actuar de una forma directa o indirecta y que impactan en la percepción del cliente en términos de calidad de la experiencia durante su estancia (Ulacia-Oviedo, 2012). Lo (2007) considera que las emociones del huésped están determinadas por la percepción que tiene de los servicios en cuanto al confort, seguridad y confiablidad. También incorpora aspectos sensoriales relacionados con los elementos tangibles e intangibles que producen en el cliente la sensación de armonía, individualidad y bienestar.

Algunos autores que han estudiado la calidad, como Gálvez y Morales (2015), Gómez, Rivera, Rodríguez y Tapia (2015) y Robledo-Galván, Meljem-Moctezuma, Fajardo-Dolci y Olvera-López (2012), consideran que no es un concepto estático ni universal y que es aplicable a muchos servicios. En el desarrollo del concepto se deben considerar distintos modelos de gestión para complementar y diseñar un método que ayude a evaluar la calidad de los servicios y mantenga los negocios en el mercado.

Los servicios de hospedaje para las ciudades que fomentan el turismo de negocios deben considerar que la calidad es fundamental para la competitividad del sector hotelero (Morillo, 2007). El buen funcionamiento de la empresa hotelera depende del conocimiento que tienen los empleados sobre las expectativas de los clientes para poder satisfacer sus necesidades.

El proceso de calidad del servicio turístico debe tener en cuenta la atención que se da al turista desde la llegada hasta su salida. Esto implica que todos los departamentos relacionados con el viajero se involucren en el proceso. También se deben incluir las industrias conexas a los servicios de hospedaje (Flores, García, Olimón y Piña, 2014).

Demanda de servicios de hospedaje

La demanda de servicios turísticos se vincula con la edad del individuo, con su nivel sociocultural y con factores como el ingreso, el nivel educativo y otros que incentivan o desincentivan el viaje (Losada, Alén y Domínguez, 2015). El género, los ingresos económicos, la capacidad de consumo y el grado de satisfacción influyen en la demanda de servicio de hoteles (Portal, Espinoza y Carreño, 2014). Las características socioeconómicas del huésped permiten al microempresario hotelero tener una mejor visión del mercado. La educación, la edad y los ingresos son elementos importantes que afectan la expectativa y percepción del visitante (Chen y Lin, 2014; Corgel, Lane y Walls, 2013).

En Ciudad del Carmen, muchos de los trabajadores del sector petrolero pagan ellos mismos su hospedaje; por lo tanto, el ingreso y el precio son factores determinantes de la demanda de hoteles. El presente estudio permitirá detectar las áreas de oportunidad para mejorar los servicios de los hoteles de tres estrellas y contribuirá a definir una estrategia adecuada para mejorar la competitividad y su posicionamiento en el mercado del sector turístico, con o sin la presencia del sector petrolero. 


\section{Metodología}

En la investigación se estudian dos aspectos: la percepción del consumidor sobre la calidad del servicio del hotel y los factores que determinan la demanda de hoteles. La percepción del consumidor se estudia a partir del modelo Servqual, y los factores que determinan dicha demanda parte del modelo clásico de demanda. El modelo Servqual consiste en recopilar, analizar y vincular datos cualitativos y cuantitativos, mientras que los modelos de demanda se ubican en el campo de la estadística inferencial.

\section{Modelo Servqual}

El modelo Servqual, que proponen Zeithaml et al. (1993), mide la expectativa, la percepción y la importancia que da el cliente a la calidad del servicio de un hotel. Esa calidad se evalúa mediante cinco dimensiones. 1) Fiabilidad: se define como la capacidad para cumplir la promesa del servicio de manera segura y precisa. 2) Capacidad de respuesta: es la voluntad de colaborar con los clientes y de prestar el servicio con prontitud. 3) Garantía: es la capacidad que tienen la empresa y sus empleados para inspirar buena voluntad y confianza en los huéspedes. 4) Empatía: consiste en trasmitir a los clientes, por medio de un servicio personalizado o adecuado, que son únicos y especiales. 5) Tangibilidad: se define como la apariencia de las instalaciones físicas, el equipo, el personal y los materiales de comunicación. ${ }^{1}$

\section{Cuestionario Servqual}

El modelo Servqual requiere el uso de un cuestionario para recabar información sobre la expectativa y la percepción del huésped. Sierra (1999) lo adaptó para analizar los servicios de alojamiento. Ésa es la versión que se utiliza en la presente investigación. Contiene 26 ítems que abarcan aspectos específicos de las cinco dimensiones antes mencionadas. Para cada ítem se evaluaron la expectativa y la percepción que tiene el huésped sobre la calidad del servicio en el hotel.

La evaluación se realizó mediante una escala de 1 a 10, donde 1 es extremadamente pobre y 10 extremadamente bueno. Una vez que el cliente cuantifica su expectativa y su percepción, por diferencia aritmética se obtiene la brecha entre ellas. Si la brecha es positiva, el cliente recibe más de lo que espera y, por lo tanto, se considera satisfecho con el servicio. Si la brecha es negativa, el huésped recibe menos de lo que espera y, por ende, no está satisfecho.

El modelo Servqual ha sido aplicado con éxito en hoteles y otras empresas de servicios, entre las que se encuentran compañías de telecomunicaciones, hospitales y cines (Basfirinci y Mitra, 2015; Matsumoto, 2014; Vergara, Quesada y Blanco, 2010). A partir del modelo Servqual, Cronin (1994) derivó el modelo Servperf, eliminando las expectativas y dejando sólo las percepciones. Este modelo analiza las dimensiones del Servqual y ha sido utilizado para analizar la calidad de diversos servicios (Ibarra-Morales y Casa-Medina, 2014). 
Validez del cuestionario Servqual

Una vez aplicado el cuestionario, se determinó su confiabilidad y su validez mediante el coeficiente alfa de Cronbach (Akbaba, 2006; Basfirinci y Mitra, 2015; Stefano, Casarotto, Barichelo y Sohn, 2015). La confiabilidad depende del número de preguntas o ítems y de la proporción de la varianza total debido a la covarianza entre sus partes (véase ecuación 1) (Ibarra-Morales, Espinoza-Galindo y Casas-Medina, 2014).

$$
\propto=\left(\frac{K}{K-1}\right)\left(1-\left(\frac{\sum_{1}^{k} S i^{2}}{S_{s u m}^{2}}\right)\right)
$$

Donde $K$ es el número de ítems de la prueba, $S i^{2}$ es la varianza de los ítems y $S_{\text {sum }}^{2}$ es la varianza de la prueba total. Cuando el valor del coeficiente es menor a 0.6 , se considera que los ítems tienen baja fiabilidad, mientras que un valor mayor a 0.6 indica una alta confiabilidad de los ítems (Matsumoto, 2014).

El coeficiente alfa de Cronbach obtenido para los datos de expectativa fue de 0.950 y para los de percepción de 0.965 (véase tabla 1); por lo tanto, el cuestionario utilizado mide ambas de manera adecuada y los datos que arroja son confiables (Akbaba, 2006; Basfirinci y Mitra, 2015; Matsumoto, 2014; Stefano et al., 2015).

Tabla 1. Validación del cuestionario y análisis factorial

\begin{tabular}{|c|c|c|}
\hline Medida/índice & Expectativa & Percepción \\
\hline Alfa de Cronbrach & 0.950 & 0.965 \\
\hline Medida Kaiser-Meyer-Olkin & 0.960 & 0.964 \\
\hline Prueba de esfericidad de Bartlett & & \\
\hline Chi-cuadrado aproximado & 9295.407 & 10663.423 \\
\hline gl & 325 & 325 \\
\hline Sig. & 0.000 & 0.000 \\
\hline
\end{tabular}

Fuente: elaboración propia con base en los datos obtenidos de las encuestas.

Análisis factorial

El análisis factorial se utilizó para encontrar grupos homogéneos de variables. Éstos se forman con las variables que se correlacionan mucho entre sí y procurando que unos grupos sean independientes de otros. Así, el análisis factorial permite saber cuáles son las variables que determinan la expectativa y la percepción del huésped. 
Para saber si es posible realizar un análisis factorial, se calculó el índice de Kaiser-Meyer-Olkin (KMO) y se empleó la prueba de esfericidad de Bartlett. El índice KMO (obtenido en la ecuación 2) compara las magnitudes de los coeficientes de correlación general o simple con respecto a las magnitudes de los coeficientes de correlación parcial (Montoya, 2007).

$$
K M O=\frac{\sum_{i \neq j} r_{i j}^{2}}{\sum_{i \neq j} r_{i j}^{2}+\sum_{i \neq j} r_{i j . m}^{2}}
$$

Donde $r_{i j}$ representa el coeficiente de correlación simple entre las variables $\mathrm{X}_{i}$ y $\mathrm{Xj}$, mientras que $r_{i j . m}$ representa la correlación parcial entre las variables $\mathrm{X} i$ y $X j$, eliminando el efecto de las restantes $m$ variables incluidas en el análisis. Cuando el modelo factorial es adecuado, la correlación parcial entre dos variables debe ser pequeña y el valor de KMO se acerca a 1 (Pérez, 2004). La medida de adecuación muestral KMO para los datos obtenidos en la expectativa y en la percepción es de 0.960 y 0.964 , respectivamente (véase tabla 1). Puesto que son cercanos a uno, se considera factible realizar el análisis factorial.

La prueba de esfericidad de Bartlett se utiliza para probar la hipótesis nula que afirma que las variables no están relacionadas en la población. Es decir, comprueba si la matriz de correlaciones es una matriz identidad. Se pueden dar como válidos aquellos resultados que presenten un valor elevado en el test y cuya fiabilidad sea menor a 0.05 . En este caso, se rechaza la hipótesis nula y conviene realizar el análisis factorial (Montoya, 2007). La prueba de esfericidad de Bartlett en este estudio es de 0.000 , lo cual asegura que el análisis factorial es adecuado para explicar los datos (véase tabla 1). El análisis de validez y el análisis factorial se realizaron con el paquete estadístico IBM SPSS.

Modelo de demanda

Nolazco y Bravo-Ortega (2015) representan el uso de sistemas de ecuaciones simultáneas en forma matricial con una estructura teórica como:

$$
Y^{\prime} T+X^{\prime} B=\mu^{\prime}
$$

Donde $Y^{\prime}$ es el conjunto de variables endógenas analizadas; $T$ la matriz que contiene los coeficientes de las variables endógenas predeterminadas; $X$ ' un vector de variables exógenas en el sistema; $B$ la matriz de coeficientes de las variables exógenas o instrumentales; y el vector del término error de cada ecuación. Labeaga y Martínez-Ros (1994) consideran que una aproximación lineal de las ecuaciones en forma estocástica se representa de la siguiente forma:

$$
\begin{aligned}
& \gamma_{1 i}=\alpha_{1} \gamma_{2 i}^{*}+\alpha_{2} \gamma_{3 i}^{*}+\beta_{1}^{\prime} X_{1 i}+u_{1 i} \\
& \gamma_{2 i}^{*}=\alpha_{3} \gamma_{1 i}+\alpha_{4} \gamma_{3 i}^{*}+\beta_{2}^{\prime} X_{2 i}+u_{2 i}
\end{aligned}
$$


Este sistema refleja decisiones óptimas de una variable endógena en términos de otra variable endógena. Moreno-Sáenz, González-Andrade y Matus-Gardea (2016) utilizan este sistema de ecuaciones porque contempla la interacción entre variables endógenas y exógenas.

En el caso de los hoteles de Ciudad del Carmen, la mayoría de los huéspedes son trabajadores foráneos que arriban a la ciudad en espera de instalarse en las plataformas petroleras. Algunos de estos trabajadores eligen el hotel debido a que las compañías no pagan su hospedaje. Por lo anterior, es posible aplicar las relaciones básicas de la teoría económica, donde la demanda de un bien depende de su precio y del ingreso de los consumidores (Chen y Lin, 2014; Chiang-Ming y Chia-Yu, 2012; Dougan, 2007; Portal, Espinoza y Carreño, 2014). Para ampliar la visión del comportamiento de los trabajadores petroleros, en el modelo se contempla el ingreso como una variable endógena que se explica por el nivel educativo y la edad del huésped (Cacciamali y Tatei, 2013; Groisman, 2014). Así, se diseñó el siguiente sistema de ecuaciones para explicar la demanda de habitaciones:

$$
\begin{aligned}
& Y=\propto_{0}+\alpha_{1} X_{1}+\alpha_{2} Z+e_{1} \\
& Z=\beta_{0}+\beta_{1} X_{2}+\beta_{2} X_{3}+e_{2}
\end{aligned}
$$

Donde $Y$ es la demanda de hoteles; $X_{1}$ el precio por día de la habitación; $Z$ el ingreso mensual de los huéspedes; $X_{2}$ los años de escolaridad; y $X_{3}$ la edad del huésped. Los estimadores del modelo se obtuvieron mediante el método de mínimos cuadrados en dos etapas. Para la estimación del modelo se utilizó el paquete estadístico Satistical Analysis System (SAS).

Población y muestra

La población de estudio son los visitantes que se hospedan en hoteles de tres estrellas. De acuerdo con el INEGI (2015), en Ciudad del Carmen se hospedaron 232105 visitantes en un hotel de tres estrellas en 2014. La muestra se calculó con un nivel de error de $5 \%$ y un nivel de confianza de $95 \%$ y se utilizó la siguiente fórmula:

$$
n=\frac{Z^{2} p q N}{(N-1) e^{2}+Z^{2} p q}
$$

Donde $\mathrm{N}=232105$ (población de estudio); $\mathrm{Z}=1.96$ (con un 95\% de nivel de confianza); $p=0.5$ (probabilidad a favor); $q=0.5$ (probabilidad en contra); $e=5 \%$ (error de estimación); y n= tamaño de la muestra, que es de 385 huéspedes.

En Ciudad del Carmen existen 138 establecimientos de hospedaje, de los cuales 27 son de tres estrellas (INEGI, 2015). La muestra se obtuvo de 16 ho- 
teles de la ciudad y para su selección se utilizó el muestreo por conveniencia, porque sólo ésos aceptaron participar en el estudio. Además, se decidió trabajar en todos los hoteles que aceptaron porque su ocupación era muy baja, y en el caso de seleccionar hoteles de manera aleatoria, obtener un número alto de entrevistas no era posible. La baja ocupación también limitó estratificar la muestra debido a que el número de entrevistas a realizar requería mucho mayor tiempo y recursos. La aplicación de los cuestionarios se realizó de febrero a agosto de 2017.

\section{Resultados}

De los huéspedes entrevistados, $89.4 \%$ visitó la ciudad por motivos laborales y de negocios, $1.5 \%$ por turismo y $9.1 \%$ por otras actividades. Lo anterior demuestra que los hoteles de tres estrellas de la ciudad dependen del sector petrolero. De dichos huéspedes, $85.8 \%$ son hombres y $14.2 \%$ mujeres. La edad promedio es de 35 años. Utilizan un hotel de la ciudad 11 veces al año y pagan 464 pesos (24 dólares) por día.

Según los datos obtenidos de la encuesta, $11.3 \%$ de los huéspedes tiene un ingreso menor a tres salarios mínimos diarios y el resto recibe más de tres. Lo anterior refleja que en el sector petrolero las remuneraciones son mayores a las del promedio nacional y esto puede ser un factor que afecta la decisión del viajero al elegir un hotel. Provienen de Tabasco $28 \%$ de los huéspedes, $25 \%$ de Veracruz, $10 \%$ de Tamaulipas y el restante 37\% proviene de Oaxaca, Chiapas, Michoacán, Quintana Roo, Yucatán, Ciudad de México y Campeche.

\section{Resultados del modelo Servqual}

En este apartado se identifican los aspectos más importantes en las expectativas y percepciones de los huéspedes, y se registran las brechas para determinar en qué aspectos del servicio se satisface al cliente.

Los resultados indican que las expectativas más altas se presentaron en las dimensiones de tangibilidad (8.06), garantía (8.03) y empatía (8.02) (véase tabla 2). La alta calificación en tangibilidad pone de manifiesto que, al llegar al hotel, el huésped espera encontrar instalaciones cómodas, agradables y funcionales. La garantía, como aspecto importante en la expectativa, señala que el huésped espera tener el cuarto disponible con los servicios ofrecidos y sentirse seguro en las instalaciones. Aunado a lo anterior, la calificación alta en empatía pone de manifiesto la necesidad de que el personal del hotel resuelva sus problemas y necesidades.

Mientras está hospedado, el huésped puede asignar una calificación al servicio que se le está proporcionando. En la columna de media percepción de la tabla 2, se puede observar que las dimensiones con mayor calificación son tangibilidad (8.14) y fiabilidad (8.14). En la dimensión de tangibilidad destaca que los huéspedes perciben instalaciones limpias, confortables y agradables. En la dimensión de fiabilidad el huésped percibe que se resuelven sus problemas 
y que los equipos del hotel funcionan de manera correcta. En el análisis, la dimensión de menor calificación es capacidad de respuesta (8.08). En esta dimensión, el huésped señala que hay poco personal para resolver sus problemas. Las variables anteriores se destacan por presentar calificaciones altas o bajas en la percepción. Sin embargo, es necesario analizar las brechas para saber si el huésped está satisfecho con los servicios.

Tabla 2. Evaluación del servicio de hoteles de tres estrellas en Ciudad del Carmen

\begin{tabular}{|c|c|c|c|c|c|c|}
\hline \multicolumn{2}{|r|}{ Dimensión/ítem } & $\begin{array}{c}\text { Media } \\
\text { expectativa }\end{array}$ & $\begin{array}{l}\text { Desviación } \\
\text { estándar }\end{array}$ & $\begin{array}{c}\text { Media } \\
\text { percepción }\end{array}$ & $\begin{array}{l}\text { Desviación } \\
\text { estándar }\end{array}$ & Brecha \\
\hline \multicolumn{2}{|r|}{ Tangibilidad } & \multicolumn{2}{|l|}{8.06} & 8.14 & \multirow[b]{2}{*}{1.59} & 0.08 \\
\hline $\mathrm{T} 1$ & $\begin{array}{l}\text { Las instalaciones y los } \\
\text { equipamientos del edificio } \\
\text { están bien conservados }\end{array}$ & 8.01 & 1.47 & 8.03 & & 0.02 \\
\hline $\mathrm{T} 2$ & $\begin{array}{l}\text { Las áreas públicas resultan } \\
\text { agradables }\end{array}$ & 8.05 & 1.45 & 8.16 & 1.48 & 0.11 \\
\hline T3 & $\begin{array}{l}\text { El personal tiene un } \\
\text { aspecto limpio y aseado }\end{array}$ & 8.00 & 1.55 & 8.02 & 1.61 & 0.02 \\
\hline T4 & Las instalaciones están limpias & 8.18 & 1.44 & 8.30 & 1.49 & 0.12 \\
\hline T5 & $\begin{array}{l}\text { Las habitaciones son confortables } \\
\text { y acogedoras }\end{array}$ & 8.07 & 1.46 & 8.21 & 1.60 & 0.14 \\
\hline \multicolumn{2}{|r|}{ Fiabilidad } & 7.97 & & 8.14 & & 0.18 \\
\hline F1 & $\begin{array}{l}\text { Se presta el servicio según las } \\
\text { condiciones pactadas }\end{array}$ & 7.94 & 1.52 & 8.09 & 1.62 & 0.15 \\
\hline F2 & $\begin{array}{l}\text { Se resuelve de forma eficaz } \\
\text { cualquier problema que } \\
\text { pueda tener el huésped }\end{array}$ & 7.98 & 1.59 & 8.24 & 3.88 & 0.27 \\
\hline F3 & Se presta el servicio sin errores & 7.84 & 1.58 & 7.95 & 1.71 & 0.11 \\
\hline F4 & $\begin{array}{l}\text { La información contenida en } \\
\text { documentos, comprobantes y } \\
\text { registros del hotel está libre } \\
\text { de errores }\end{array}$ & 8.12 & 1.43 & 8.21 & 1.57 & 0.09 \\
\hline F5 & $\begin{array}{l}\text { Los equipamientos funcionan cor- } \\
\text { rectamente (ascensores, luces, elec- } \\
\text { trodomésticos y medios } \\
\text { audiovisuales) }\end{array}$ & 7.96 & 1.40 & 8.23 & 1.44 & 0.27 \\
\hline \multicolumn{2}{|r|}{ Capacidad de respuesta } & 7.97 & & 8.08 & & 0.11 \\
\hline C1 & $\begin{array}{l}\text { Se consigue fácilmente cualquier } \\
\text { información cuando la necesita }\end{array}$ & 7.98 & 1.53 & 8.17 & 1.63 & 0.19 \\
\hline $\mathrm{C} 2$ & $\begin{array}{l}\text { Los diferentes servicios se realizan } \\
\text { con rapidez }\end{array}$ & 7.98 & 1.49 & 8.12 & 1.69 & 0.13 \\
\hline C3 & $\begin{array}{l}\text { El personal está dispuesto } \\
\text { a ayudar a los huéspedes }\end{array}$ & 8.00 & 1.48 & 8.02 & 1.57 & 0.02 \\
\hline $\mathrm{C} 4$ & $\begin{array}{l}\text { Siempre hay personal disponible } \\
\text { para proporcionar al huésped la } \\
\text { información cuando la necesita }\end{array}$ & 7.89 & 1.54 & 8.02 & 1.58 & 0.13 \\
\hline
\end{tabular}




\begin{tabular}{|c|c|c|c|c|c|c|}
\hline C5 & $\begin{array}{l}\text { Siempre hay alguna persona de la } \\
\text { Dirección a disposición del huésped } \\
\text { para cualquier problema que pueda } \\
\text { surgir }\end{array}$ & 8.00 & 1.53 & 8.07 & 1.56 & 0.07 \\
\hline \multicolumn{2}{|r|}{ Garantía } & 8.03 & & 8.10 & & 0.07 \\
\hline G1 & El personal genera confianza & 7.81 & 1.60 & 7.97 & 1.61 & 0.16 \\
\hline G2 & $\begin{array}{l}\text { Se actúa con discreción y } \\
\text { se respeta la intimidad del huésped }\end{array}$ & 8.03 & 1.46 & 8.14 & 1.54 & 0.11 \\
\hline G3 & $\begin{array}{l}\text { El personal es competente } \\
\text { y profesional }\end{array}$ & 8.07 & 4.81 & 8.03 & 1.67 & 0.04 \\
\hline G4 & $\begin{array}{l}\text { Las instalaciones son seguras } \\
\text { (cumplen las normas de seguridad) }\end{array}$ & 8.21 & 1.50 & 8.28 & 1.59 & 0.07 \\
\hline \multicolumn{2}{|r|}{ Empatía } & 8.02 & & 8.10 & & 0.08 \\
\hline E1 & $\begin{array}{l}\text { Se proporciona una atención } \\
\text { individualizada }\end{array}$ & 8.02 & 1.77 & 8.03 & 1.80 & 0.01 \\
\hline E2 & $\begin{array}{l}\text { El personal se dirige al huésped } \\
\text { por su nombre, se presta un servicio } \\
\text { personalizado }\end{array}$ & 8.03 & 1.83 & 8.05 & 1.95 & 0.02 \\
\hline E3 & $\begin{array}{l}\text { El personal se esfuerza por conocer } \\
\text { las necesidades de cada huésped }\end{array}$ & 7.82 & 1.70 & 7.94 & 1.84 & 0.11 \\
\hline E4 & $\begin{array}{l}\text { El huésped es lo más importante (lo } \\
\text { primero son los intereses } \\
\text { de los huéspedes) }\end{array}$ & 7.97 & 1.66 & 8.14 & 1.62 & 0.17 \\
\hline E5 & $\begin{array}{l}\text { Los horarios de los diferentes } \\
\text { servicios se adaptan a las } \\
\text { necesidades del huésped }\end{array}$ & 8.05 & 1.49 & 8.11 & 1.68 & 0.05 \\
\hline E6 & $\begin{array}{l}\text { Los accesos al hotel son fáciles } \\
\text { (su localización, estacionamiento, } \\
\text { señalética, etc) }\end{array}$ & 8.16 & 1.39 & 8.26 & 1.58 & 0.10 \\
\hline E7 & $\begin{array}{l}\text { Los empleados se preocupan por } \\
\text { resolver los problemas del huésped }\end{array}$ & 8.09 & 1.59 & 8.15 & 1.69 & 0.06 \\
\hline
\end{tabular}

Fuente: elaboración propia con base en las encuestas.

Al calcular las brechas, se observa que los promedios más bajos los obtuvieron las dimensiones de garantía (0.07), tangibilidad $(0.08)$ y empatía $(0.08)$. Dentro de la dimensión de garantía, destaca un ítem con brecha negativa que indica que el personal no es competente ni profesional. En la dimensión de tangibilidad, las brechas más bajas se presentaron en la limpieza del personal y en el estado físico de las instalaciones y de los equipos del hotel.

La dimensión de empatía también presentó una de las brechas más bajas. En esta dimensión, los ítems con brechas más bajas hacen referencia a la atención que el trabajador le proporciona al huésped. En específico, hay dificultad por parte de los empleados para proporcionar una atención personalizada y demostrar su preocupación por resolver los problemas del cliente. Las brechas calculadas para esta dimensión sobresalen debido a que presentan las expectativas más altas; por lo tanto, los hoteles deben mejorar estos servicios para dejar satisfecho al huésped. 
Las brechas en todos los demás ítems son positivas pero muy cercanas a cero, y por la desviación estándar (véase tabla 2), es posible inferir que una cantidad considerable de los huéspedes entrevistados no está satisfecha con el servicio. Por lo tanto, es posible afirmar que los hoteles de tres estrellas de la ciudad presentan dificultades para satisfacer las expectativas de los huéspedes.

Resultados del análisis factorial

Se realizó el análisis factorial para obtener grupos homogéneos de datos llamados factores. En éstos se identificaron los ítems con las comunalidades más altas. La comunalidad mide la correlación que existe entre cada ítem con el resto de los ítems del cuestionario aplicado. De acuerdo con Lloret-Segura, Ferreres-Tracer, Hernández-Baeza y Tomás-Marcos (2014), una condición óptima se presenta cuando las comunalidades son mayores a 0.7 . A continuación, se registran los ítems más importantes de cada factor en la expectativa y percepción, tomando en cuenta una comunalidad superior a 0.8 .

\section{Análisis factorial para las expectativas}

En el análisis factorial se encontraron tres factores para las expectativas del cliente. En el factor 1, los resultados de cuatro ítems de la dimensión de capacidad de respuesta son los que presentan comunalidades más altas y determinan si el huésped espera recibir un servicio adecuado y con rapidez. En el factor 2, todas las respuestas de la dimensión de tangibilidad tienen las comunalidades más altas; por lo tanto, el huésped espera que las instalaciones, el equipo y los muebles del hotel estén bien conservados y limpios. En el factor 3, las comunalidades más altas se presentan en dos de las respuestas de la dimensión de empatía, que consisten en la facilidad de acceso al hotel, el horario de servicios y la seguridad de las instalaciones.

Tabla 3. Matriz de componentes rotados, 2018

\begin{tabular}{|c|c|c|c|c|c|c|}
\hline & \multirow{2}{*}{ Dimensión/ítem } & \multicolumn{3}{|c|}{ Expectativa } & \multicolumn{2}{|c|}{ Percepción } \\
\hline & & 1 & 2 & 3 & 1 & 2 \\
\hline \multicolumn{7}{|c|}{ Tangibilidad } \\
\hline T11 & $\begin{array}{l}\text { Las instalaciones y los equipamientos } \\
\text { del edificio están bien conservados }\end{array}$ & & 0.756 & & & 0.820 \\
\hline T21 & Las áreas públicas resultan agradables & & 0.766 & & & 0.819 \\
\hline T31 & El personal tiene un aspecto limpio y aseado & & 0.803 & & & 0.805 \\
\hline T41 & Las instalaciones están limpias & & 0.779 & & & 0.832 \\
\hline T51 & Las habitaciones son confortables y acogedoras & & 0.799 & & & 0.843 \\
\hline \multicolumn{7}{|c|}{ Fiabilidad } \\
\hline F11 & Se presta el servicio según las condiciones pactadas & & 0.745 & & & 0.754 \\
\hline
\end{tabular}




\begin{tabular}{|c|c|c|c|c|c|c|}
\hline F21 & $\begin{array}{l}\text { Se resuelve de forma eficaz cualquier problema } \\
\text { que pueda tener el huésped }\end{array}$ & & & & & \\
\hline F31 & Se presta el servicio sin errores & & 0.704 & & & \\
\hline F41 & $\begin{array}{l}\text { La información contenida en documentos, } \\
\text { comprobantes y registros del hotel está libre de } \\
\text { errores }\end{array}$ & & & & & 0.764 \\
\hline F51 & $\begin{array}{l}\text { Los equipamientos funcionan correctamente } \\
\text { (ascensores, luces, electrodomésticos, } \\
\text { y medios audiovisuales) }\end{array}$ & & & & & \\
\hline \multicolumn{7}{|c|}{ Capacidad de respuesta } \\
\hline C11 & $\begin{array}{l}\text { Se consigue fácilmente cualquier información } \\
\text { cuando la necesita }\end{array}$ & 0.786 & & & 0.769 & \\
\hline C21 & Los diferentes servicios se realizan con rapidez & 0.802 & & & 0.778 & \\
\hline C31 & El personal está dispuesto a ayudar a los huéspedes & 0.761 & & & & \\
\hline C41 & $\begin{array}{l}\text { Siempre hay personal disponible para proporcionar } \\
\text { al huésped la información cuando la necesita }\end{array}$ & 0.720 & & & 0.741 & \\
\hline C51 & $\begin{array}{l}\text { Siempre hay alguna persona de la Dirección a } \\
\text { disposición del huésped para cualquier problema } \\
\text { que pueda surgir }\end{array}$ & & & & 0.777 & \\
\hline \multicolumn{7}{|c|}{ Garantía } \\
\hline G11 & El personal genera confianza & & & & 0.759 & \\
\hline G21 & $\begin{array}{l}\text { Se actúa con discreción y se respeta la intimidad } \\
\text { del huésped }\end{array}$ & & & & & \\
\hline G31 & El personal es competente y profesional & & & & 0.808 & \\
\hline G41 & $\begin{array}{l}\text { Las instalaciones son seguras (cumplen las normas } \\
\text { de seguridad) }\end{array}$ & & & 0.724 & 0.706 & \\
\hline \multicolumn{7}{|c|}{ Empatía } \\
\hline E11 & Se proporciona una atención individualizada & 0.702 & & & 0.838 & \\
\hline E21 & $\begin{array}{l}\text { El personal se dirige al huésped por su nombre, } \\
\text { se presta un servicio personalizado }\end{array}$ & & & & 0.807 & \\
\hline E31 & $\begin{array}{l}\text { El personal se esfuerza por conocer las necesidades } \\
\text { de cada huésped }\end{array}$ & & & & 0.843 & \\
\hline E41 & $\begin{array}{l}\text { El huésped es lo más importante } \\
\text { (lo primero son los intereses de los huéspedes) }\end{array}$ & & & & 0.799 & \\
\hline E51 & $\begin{array}{l}\text { Los horarios de los diferentes servicios } \\
\text { se adaptan a las necesidades del huésped }\end{array}$ & & & 0.783 & 0.763 & \\
\hline E61 & $\begin{array}{l}\text { Los accesos al hotel son fáciles (su localización, } \\
\text { estacionamiento, señalética, etc) }\end{array}$ & & & 0.839 & & \\
\hline E71 & $\begin{array}{l}\text { Los empleados se preocupan por resolver } \\
\text { los problemas del huésped }\end{array}$ & & & & 0.807 & \\
\hline & Porcentaje de varianza explicada & 25.37 & 24.62 & 18.76 & 41.47 & 27.10 \\
\hline
\end{tabular}

Fuente: elaboración propia con base en las encuestas. 
Análisis factorial para la percepción

En el análisis factorial para la percepción se simplificó el cuestionario a las dos dimensiones más importantes: la tangibilidad y la empatía. Y es que la percepción de los huéspedes sobre la calidad del servicio depende de dos aspectos: la atención que le proporcionan los empleados del hotel y la apariencia de las instalaciones.

En el factor 1, los ítems con comunalidades más altas determinan si el personal se esfuerza por conocer las necesidades y resolver los problemas del huésped, si se le proporciona un servicio personalizado y si el hotel es seguro y de fácil localización.

En el factor 2, las variables más importantes son las relacionadas con la tangibilidad. Es decir, para que el huésped esté satisfecho es necesario que las instalaciones y los equipos estén bien conservados, que las áreas comunes sean agradables, que el personal tenga un aspecto limpio y aseado y que las instalaciones se encuentren limpias.

\section{Resultados del modelo de regresión}

Los resultados del modelo de ecuaciones simultáneas muestran una relación inversa entre la demanda de servicios de hotel y el precio por habitación (véase tabla 4). El coeficiente de la elasticidad precio (0.15) indica que la demanda de habitaciones es poco sensible ante los cambios en el precio. Un aumento de $1 \%$ en el precio provoca una disminución de $0.15 \%$ en la demanda de habitaciones.

En Ciudad del Carmen, la elasticidad ingreso de la demanda (0.63) muestra una relación inversa entre el ingreso de los huéspedes y la demanda de habitaciones. A medida que aumenta en $1 \%$ el ingreso de éstos, la demanda de cuartos de hoteles de tres estrellas disminuye en $\mathbf{0 . 6 3 \%}$.

Tabla 4. Resultados del modelo de regresión

\begin{tabular}{|c|c|c|c|}
\hline Variable & Estimador & $\operatorname{Pr}>|t|$ & Elasticidad \\
\hline \multicolumn{4}{|c|}{ Función de demanda } \\
\hline Intercepto & 16.147 & $<.0001$ & \\
\hline Precio de la habitación (X1) & 0.00413 & 0.1024 & 0.15 \\
\hline \multirow[t]{2}{*}{ Ingreso del huésped ( $Z$ ) } & 0.00079 & 0.0038 & 0.63 \\
\hline & $\operatorname{Pr}>F=0.0001 ; R^{2}=0.08$ & & \\
\hline \multicolumn{4}{|c|}{ Función de ingreso } \\
\hline Intercepto & 270.7280 & 0.8255 & \\
\hline Años de escolaridad & 516.4031 & $>.0001$ & 0.68 \\
\hline \multirow[t]{2}{*}{ Edad } & 83.16033 & 0.0004 & 0.29 \\
\hline & $\operatorname{Pr}>F=0.0001 ; R^{2}=0.20$ & & \\
\hline
\end{tabular}

Fuente: elaboración propia con datos de las encuestas. 
Los coeficientes de elasticidad permiten observar que la demanda de habitaciones es más sensible a los cambios en los ingresos de los huéspedes que a su precio. La relación inelástica entre el precio y la demanda se debe a que las compañías realizan convenios y pagan las habitaciones a una parte de los trabajadores encuestados. Por otro lado, cuando su ingreso aumenta, los trabajadores petroleros tienden a sustituir los hoteles de tres estrellas por hoteles de mejor calidad, por lo general hoteles de alguna cadena.

El modelo de regresión también explica por qué las remuneraciones en el sector petrolero son altas. Los coeficientes estimados para el ingreso en función de la escolaridad (0.68) y la edad (0.29) muestran que, a mayor edad y escolaridad de los trabajadores, mayores son sus salarios. Al respecto, $33 \%$ de los encuestados tiene licenciatura, $32 \%$ cuenta con estudios de nivel bachillerato, $9 \%$ carrera técnica, $15 \%$ primaria y secundaria y $11 \%$ no contestó. Lo anterior muestra que el sector petrolero exige mano de obra calificada y por ello sus remuneraciones son más altas que el promedio nacional.

\section{Discusión}

De acuerdo con el modelo Servqual, los huéspedes de los hoteles tienen altas expectativas sobre el estado físico de las instalaciones (tangibilidad) y sobre la atención personal que puedan recibir (empatía). Aunque las expectativas representan el rendimiento esperado del servicio (Laufer, 2002) basados en la experiencia previa (Álvarez, Fraiz y Río, 2013; Zeithaml, Berry y Parasuraman, 1993), es importante tomarlas en cuenta, porque se utilizan como referencia al evaluar el desempeño de la empresa (Choi y Mattila, 2008).

En las percepciones de los huéspedes, las dimensiones de garantía y de fiabilidad son importantes. En el sector turístico la calidad percibida afecta la intención de recomendación y la intención de visita, al tiempo que disminuye la sensibilidad al precio (González, Rodríguez y Fraiz, 2007). Aunque estos huéspedes utilizan los hoteles por motivos laborales, es importante centrar la atención en el mejoramiento de las instalaciones para que puedan recomendarlos.

En todas las dimensiones las brechas calculadas son cercanas a cero. Morillo y Morillo (2016) encontraron el mismo resultado para los hoteles de entre una y cuatro estrellas. Sánchez, Sánchez y Hernández (2010) también encontraron que la diferencia entre expectativa y percepción es cercana a cero en los hoteles de cinco estrellas. Lo anterior muestra la necesidad de mejorar la calidad de los servicios, independientemente de la categoría y del lugar en que se encuentren los hoteles.

Las dimensiones de garantía, empatía y tangibilidad obtuvieron las menores brechas; por lo tanto, los hoteles deben centrar su atención en el mejoramiento de estas áreas. Ríos, Ortega y Matilla (2016) también concluyen que las habitaciones, el trato y la eficacia del personal son los factores más valorados por los huéspedes. Por ende, a escala global y sin importar el país donde se ubique el hotel, los clientes consideran que la eficacia del personal, el estado físico 
de las instalaciones y la atención personalizada son los factores que permiten hacer placentera su estancia.

Importancia de las expectativas y de las percepciones

El análisis factorial muestra que en las expectativas y percepciones de los huéspedes las dimensiones de empatía y tangibilidad son las más importantes. Y en el caso de la expectativa en particular, la capacidad de respuesta es muy significativa. Los aspectos relacionados con la decoración, la limpieza, el estado de las habitaciones y la cortesía del personal son cualidades que valoran los clientes y determinan su satisfacción (Gundersen, Heide y Olsson, 1996; Ramsaran-Fowdar, 2006). Por eso, para aumentar la calidad de los servicios, los hoteles deben centrar su atención en mejorar sus instalaciones y la atención al huésped.

El análisis factorial muestra que los visitantes no llegan con la expectativa de recibir un servicio personalizado (empatía), pero después este factor cobra importancia. El servicio que perciben los huéspedes de los hoteles influirá en la duración de su visita, en las posibilidades de su retorno y en la recomendación a sus familiares y amigos (Douglas y Bateson, 2012). La empatía es fundamental para el crecimiento del sector hotelero y del turismo (Scheyvens y Russell, 2012). En este sentido, la calidad del servicio está ligada a la satisfacción del cliente (González, Frías-Jiménez y Gómez-Figueroa, 2016; Morillo y Morillo, 2016; Vergara, Quesada y Blanco, 2011). Entonces, es indispensable que los hoteles desarrollen políticas de capacitación constante del personal. La capacitación debe fomentar el desarrollo de las habilidades interpersonales de los trabajadores para que sean corteses, amigables, competentes y capaces de resolver los problemas y atender las necesidades del huésped (Ramsaran-Fowdar, 2007).

El precio y el ingreso como determinantes de la demanda de hospedaje

En el presente estudio se obtuvo una relación inelástica entre el precio y la demanda de hoteles. Otros estudios también estiman que los visitantes no dan mucha importancia al precio de las habitaciones (Chiang-Ming y Chia-Yu, 2012; Dougan, 2007; Portal et al., 2014). Al respecto, la duración de la estadía es un factor importante en la relación entre precio y demanda (Masiero y Nicolau, 2012). Los huéspedes en Ciudad del Carmen utilizan los hoteles en periodos de uno o dos días y esto puede explicar la relación inelástica entre el precio y el consumo.

El ingreso de los consumidores también influye en la demanda de hoteles (Corgel et al., 2013). Los resultados muestran una relación inversa entre el ingreso del huésped y la demanda de hoteles. Sin embargo, es muy diferente al reportado por Chen y Lin (2014), quienes encontraron un efecto positivo del ingreso sobre la demanda de hoteles. En el turismo recreativo, el ingreso desempeña un papel fundamental (Dougan, 2007), mientras que en el turismo de negocios el precio y el ingreso tienen menor importancia. 
El efecto inverso entre el ingreso y la demanda de hoteles de tres estrellas se puede explicar por los altos salarios de los huéspedes entrevistados. En las características socioeconómicas se muestra que éstos tienen una escolaridad alta y los resultados del modelo de regresión muestran que a mayor escolaridad, mayor es el ingreso de los huéspedes. Cacciamali y Tatei (2013) y Groisman (2014) también encontraron que la educación determina el salario de los trabajadores. Por lo tanto, es posible asumir que, en el caso de los trabajadores de la industria petrolera, éstos reciben mejores salarios debido al grado de calificación que tienen.

Los trabajadores con altos ingresos pueden hospedarse en hoteles de mayor categoría y mayor precio. Por consiguiente, los hoteles de tres estrellas de la ciudad se enfrentan a un mercado más exigente de la calidad de los servicios, porque los huéspedes tienen la capacidad de sustituir un hotel de tres estrellas por uno de cuatro o cinco estrellas.

\section{Conclusiones}

En Ciudad del Carmen, la expectativa de los huéspedes es que los hoteles tengan instalaciones, equipos y muebles limpios y en buen estado, que se les atienda con calidez y que el personal sea capaz de adaptarse a sus necesidades. Pero una vez hospedados, la percepción que tienen sobre la calidez de los empleados y los aspectos físicos del hotel no es del todo buena. Como resultado, las brechas entre las expectativas y las percepciones sobre estos aspectos son positivas pero muy cercanas a cero. La misma situación se repite en cada uno de los ítems de las cinco dimensiones del modelo Servqual, lo que pone en evidencia la dificultad de los hoteles para dejar a los huéspedes satisfechos con los servicios que ofrecen.

En Ciudad del Carmen, cerca de $90 \%$ de los huéspedes son trabajadores del sector petrolero que pernoctan en la ciudad para después subir a las plataformas o asistir a capacitación. La relación entre precio y consumo es inelástica y el ingreso de los huéspedes afecta de manera inversa la demanda de hoteles de tres estrellas. La relación inelástica entre el precio y el consumo se debe a que una parte de los trabajadores no paga por las habitaciones. Los que lo hacen, sólo utilizan los hoteles para pernoctar. El sector hotelero fue uno de los más perjudicados por la contracción de la actividad petrolera y los cambios en la política energética. Ante la caída de los niveles de ocupación, la competencia basada en precios resulta inadecuada debido a que la relación entre precio y consumo es inelástica, y la disminución de los precios implica una disminución mayor en los ingresos del hotel.

La relación negativa entre la demanda de habitaciones y el ingreso de los trabajadores petroleros se debe a su alto poder adquisitivo, lo cual les permite tener la opción de hospedarse en hoteles de mayor categoría. De este modo, los hoteles de tres estrellas de la ciudad tienen una fuerte competencia con hoteles de cuatro o cinco estrellas, cuya calidad de servicios debe ser mejor. 
La fuerte demanda de mercado que presentó este sector durante el auge petrolero no permitió a los empresarios calcular el ciclo de vida de la industria. Por eso no realizaron una planeación estratégica a mediano y largo plazos. No mejoraron sus instalaciones ni la calidad de la atención al huésped. Un cambio de estrategia que lleve a un mejoramiento de las instalaciones y de la atención del personal, contribuirá a que los huéspedes perciban que los hoteles de tres estrellas proporcionan un servicio de buena calidad, lo cual se reflejará en una mejor competitividad. Aunado a lo anterior, si los demás servicios de la ciudad son también de buena calidad y se cuenta con una vasta oferta turística, los visitantes percibirán que Ciudad del Carmen es una opción turística y los hoteles no dependerán sólo del sector petrolero.

\section{Referencias}

Akbaba, A. (2006). Measuring service quality in the hotel industry: a study in a business hotel in Turkey. Hospitality Management, 25, 170-192. doi: 10.1016/j.ijhm.2005.08.006

Álvarez, J., Fraiz, J. A., y Río, M. C. del. (2013). Q de calidad turística. Barreras a la implantación y certificación. Cuadernos de Turismo, 32, 11-35. Recuperado de https: / / revistas.um.es/turismo/article/view/177391/149091

Basfirinci, C., y Mitra, A. (2015). A cross cultural investigation of airlines service quality through integration of Servqual and the Kano model. Journal of Air Transport Management, 42(1), 239-248. doi: 10.1016/j.jairtraman.2014.11.005

Blancas, D. (2016). (30 de marzo de 2016). Se extinguen los hoteles en Ciudad del Carmen; no ven opciones. Crónica. Recuperado de https:/ / www.cronica. com.mx/notas/2016/952632.html

Cacciamali, M. C., y Tatei, F. (2013). Género y salarios de la fuerza de trabajo calificada en Brasil y México. Problemas del Desarrollo, 172(44), 53-79. doi: 10.22201/iiec.20078951e.2013.172.34557

Castillo, R. del, López-Mateo, C., y Sánchez-Fernández, M. D. (2019). Responsabilidad social municipal bajo la percepción de los jóvenes en ciudades de turismo de negocios y recreativo. Revista Administracao em Diálogo, 22(1), 57-80. doi: 10.23925/2178-0080.2020v22i1.41162

Chen, C.-M., y Lin, Y.-C. (2014). The effect of weather on the demand for rooms in the Taiwanese hotel industry: an examination. Tourism Management Perspectives, 12, 81-87. doi: 10.1016/j.tmp.2014.09.004

Chiang-Ming, C., y Chia-Yu, Y. (2012). The causality examination between demand uncertainty and hotel failure: a case study of international tourist hotels in Taiwan. International Journal of Hospitality Management, 31, 10451049. doi: 10.1016/j.ijhm.2011.12.011

Choi, S., y Mattila, A. S. (2008). Perceived controllabity and service expectations: influences on customer reaction following service failure. Journal of Business Research, 61(1), 24-30. doi: 10.1016/j.jbusres.2006.05.006 
Confederación de Cámaras Nacionales de Comercio, Servicios y Turismo (CONCANACO-SERVYTUR). (Enero de 2016). Boletín Turismo. Recuperado de http: / / www.concanaco.com.mx/wp-content/uploads/2016/boletines/Turismo-Enero-2016.pdf

Corgel, J., Lane, J., y Walls, A. (2013). How currency exchange rates affect the demand for U. S. hotel rooms. International Journal of Hospitality Management, 35, 78-88. doi: 10.1016/j.ijhm.2013.04.014

Cronin, J. (1994). Servperf vs. Servqual: reconciling performance based and perceptions minus expectations measurement of service quality. Journal of Marketing, 58(1), 125-131. doi: 10.1177/002224299405800110

Dougan, J. W. (2007). Analysis of Japanese tourist demand to Guam. Asia Pacific of Tourism Research, 12(2), 79-88. doi: 10.1080/10941660701243307

Douglas, K., y Bateson, J. E. G. (2012). Marketing de servicios: conceptos, estrategias y casos. Ciudad de México: Cengage Learning.

Flamarich, M., y Duro, J. A. (2011). Turismo de negocios (eventos) en España. Análisis y prespectivas. Papers de Turismo, (49-50), 59-74. Recuperado de http://www. papersdeturisme.gva.es/ojs/index.php/Papers/article/viewFile/168/147

Flores, D. A., García, D. del C., Olimón, A. Y., y Piña, M. F. (2014). La importancia de las relaciones humanas para la calidad en el servicio turístico. Revista Educateconciencia, 4(5), 6-14. Recuperado de http://tecnocientifica.com. $\mathrm{mx} /$ educateconciencia/index.php/revistaeducate/article/view/67

Freyle, R. A. (2010). Las MIPYMES colombianas, escenario estratégico para la creatividad e innovación del talento humano. Escenarios, 8(2), 31-39. Recuperado de http://hdl.handle.net/11619/1636

Gálvez, R. P., y Morales, S. V. (2015). Desarrollo y validación del cuestionario para la evaluación de la calidad percibida en servicios deportivos. Cultura, ciencia y deporte: revista de ciencias de la actividad física y del deporte de la Universidad Católica de San Antonio, 28(01), 55-66. Recuperado de https: / / www.redalyc.org/service/r2020/downloadPdf/1630/163036903003/6

Gómez, S., Rivera, A. G., Rodríguez, M., y Tapia, A. (2015). Un modelo para la recolección de indicadores de calidad en los servicios gerontológicos. Enseñanza e Investigación en Psicología, 20(1), 56-68. Recuperado de https:// www. redalyc.org/pdf/292/29242798009.pdf

González, M. E. A., Rodríguez, L., y Fraiz, J. A. (2007). Assessing tourist behavioral intentions through perceived service quality and customer satisfaction. Journal of Business Research, 60(2), 153-160. doi: 10.1016/j.jbusres.2006.10.014

González, M., Frías-Jiménez, R., y Gómez-Figueroa, O. (2016). Análisis de la calidad percibida por el cliente en la actividad hotelera. Ingeniería Industrial, 37(3), 253-265. Recuperado de https://www.redalyc.org/articulo. oa?id=360448031004

González, S. R. (2011). La innovación abierta en las empresas turísticas extremeñas como herramienta de entrada en el turismo de negocios. Pasos: Revista de Turismo y Patrimonio Cultural, 9(4), 489-502. doi: 10.25145/j. pasos.2011.09.049 
Groisman, F. (2014). Empleo, salarios y desigualdad en Argentina: análisis de los determinantes distributivos. Revista Problemas del Desarrollo, 177(45), 59-86. doi: 10.1016/S0301-7036(14)70863-6

Grönroos, C. (1994). Marketing y gestión de servicios. La gestión de los momentos de la verdad y la competencia en los servicios. Madrid: Ediciones Díaz de Santos, S. A.

Grzinic, J. (2007). Concepts of service quality measurement in hotel industry. Ekon. Misao Praksa DBK, 16, 81-98. Recuperado de http://eprints.undip. ac.id/3396/

Gundersen, M. G., Heide, M., y Olsson, U. H. (1996). Hotel guest satisfaction among business travelers: What are the important factors? The Cornell Hotel and Restaurant Administration Quarterly, 37(2), 72-81. doi: 10.1016/00108804(96)83104-1

Hinojosa, A., y Martínez, E. (2018). La patrimonialización del Parque La Fundidora en Monterrey, Nuevo León. Anuario Humanitas, 45(1), 141-159. Recuperado de http://www.humanitas.uanl.mx/index.php/ah/article/view/218

Ibarra-Morales, L. E., y Casas-Medina, E. V. (2014). Aplicación del modelo Servperf en los centros de atención Telcel, Hermosillo: una medición de la calidad en el servicio. Contaduría y Administración, 60(1), 229-260. doi: 10.1016/S0186-1042(15)72153-4

Ibarra-Morales, L. E., Espinoza-Galindo, B. E., y Casas-Medina, V. (2014). Aplicación del modelo Servqual para evaluar la calidad en el servicio en los hospitales públicos de Hermosillo, Sonora. Tecnociencia, 8(2), 98-108. Recuperado de http:/ / tecnociencia.uach.mx/numeros/v8n2/data/Aplicacion_ del_modelo_Servqual_para_evaluar_la_calidad_en_el_servicio_en_los_hospitales_publicos_de_Hermosillo_Sonora.pdf

Instituto Nacional de Estadística y Geografía (INEGI). (2012). Anuario Estadístico de Campeche.

Instituto Nacional de Estadística y Geografía (INEGI). (2014). Los municipios más importantes en México: visión censal. Censos económicos, 2014. Recuperado de http://www.beta.inegi.org.mx/contenidos/proyectos/ce/2014/ doc/minimonografias/m_dmmip_ce2014.pdf

Instituto Nacional de Estadística y Geografía (INEGI). (2015). Anuario Estadístico del Estado de Campeche.

Instituto Nacional de Estadística y Geografía (INEGI). (2017). Anuario Estadístico y Geográfico de los Estados Unidos Mexicanos.

Labeaga, J. M., y Martínez-Ros, E. (1994). Estimación de un modelo de ecuaciones simultáneas con variables dependientes limitadas: una aplicación con datos de la industria española. Investigaciones Económicas, 18(3), 465-489. Recuperado de http://hdl.handle.net/10016/7912

Laufer, D. (2002). Are antecedents of consumer dissatisfaction and consumer attributions for product failures universal? Advances in Consumer Research, 29(1), 312-317. Recuperado de https: / /www.acrwebsite.org/volumes/8665

Lloret-Segura, S., Ferreres-Tracer, A., Hernández-Baeza, A., y Tomás-Marcos, I. (2014). El análisis factorial exploratorio de los ítems: una guía práctica, revisada y actualizada. Anales de Psicología, 30(3), 1151-1169. doi: 10.6018/ analesps.30.3.199361 
Lo, K. P. Y. (2007). Emotional design for hotel stay experiences: research on guest emotions and design opportunities. International Association of Societies of Design Research. Hong Kong Polytechnic University. Recuperado de https: / / www.sd.polyu.edu.hk/iasdr/proceeding/papers/Emotional\%20design\%20for\%20hotel\%20stay\%20experiences_\%20Research\%20on\%20guest\%20 emotions\%20and\%20design\%20opportunities.pdf

López-Rodríguez, A. L. (2009). Sistema integrado de gestión para empresas turísticas. Estudios Gerenciales, 25(112), 131-150. doi: 10.1016/S01235923(09)70083-X

Losada, S. M. N., Alén, G. M. E., y Domínguez, V. T. (2015). Análisis de los determinantes de la decisión de viajar de los senior españoles. Estudios y Perspectivas en Turismo, 24(1), 1-20. Recuperado de https://dialnet.unirioja. es/servlet/articulo?codigo=5205729

Macdonald, E. K., Wilson, H., Martínez, V., y Toossi, A. (2011). Assessing value-in use: a conceptual framework and exploratory study. Industrial Marketing Management, 40(5), 671-682. doi: 10.1016/j.indmarman.2011.05.006

Marchante, A. J., y Ortega, B. (2010). Capital humano, desajuste educativo y productividad del trabajo: un estudio para la industria hotelera. Cuadernos de Economía y Dirección de la Empresa, 44, 79-100. doi: 10.1016/S11385758(10)70020-7

Marques, J., y Santos, N. (2017). Desarrollo del turismo de negocios: una perspectiva portuguesa. Cuadernos de Turismo, 40, 423-437. Recuperado de https: / / dialnet.unirioja.es/servlet/articulo?codigo=6271453

Martín, E. M. (2011). El turismo de negocios: una actividad en auge. Espacios y destinos turísticos en tiempos de globalización y crisis. Madrid: Universidad Carlos III. Recuperado de https: / / core.ac.uk/download/pdf/29403942.pdf

Masiero, L., y Nicolau, J. L. (2012). Price sensitivity to tourism activities: looking for determinant factors. Tourism Economics, 18(4), 675-690. doi: $10.5367 /$ te.2012.0143

Matsumoto, R. (2014). Desarrollo del modelo Servqual para la medición de la calidad del servicio en la empresa de publicidad Ayuda Experto. Perspectivas, 34, 181-209. Recuperado de https: / /www.redalyc.org/pdf/4259/425941264005. pdf

Mayo, A., Guzmán, A., y Luna, L. I. (2016). Tabasco en la necesaria convergencia de sus proyectos de inversión en el turismo de negocios y de ocio. International Journal of Scientific Management and Tourism, 2(1), 355-370. Recuperado de https: / / dialnet.unirioja.es/servlet/articulo?codigo=5665895

Monfort, M., Defante, L. R., Oliveira, D. de, y Mantovani, D. (2013). Satisfacción del consumidor de servicios hoteleros: implicaciones estratégicas. Estudios y Perspectivas en Turismo, 22(2), 276-293. Recuperado de https: / / dialnet.unirioja.es/servlet/articulo?codigo $=5131426$

Montoya, O. (2007). Aplicación del análisis factorial a la investigación de mercados. Caso de estudio. Scientia et Technical, 13(35), 281-286. doi: $10.22517 / 23447214.5443$

Moreno-Sáenz, L. I., González-Andrade, S., y Matus-Gardea, J. A. (2016). Dependencia de México a las importaciones de maíz en la era del TLCAN. Re- 
vista Mexicana de Ciencias Agrícolas, 7(1), 115-126. doi: 10.29312/remexca. v7i1.375

Morillo, M. C. (2007). Análisis de la calidad del servicio hotelero mediante la Escala de Servqual. Caso hoteles de turismo del municipio Libertador del estado Mérida. Visión Gerencial, 6(2), 269-297. Recuperado de http://iies. faces.ula.ve/Revista/Articulos/Revista_27/Pdf/Rev27Morillo.pdf

Morillo, M. C. (2011). Turismo y producto turístico. Evolución, conceptos, componentes y clasificación. Visión Gerencial, 1(1), 135-158. Recuperado de http: / / www. redalyc.org / articulo.oa?id=465545890011

Morillo, M., y Morillo, M. del C. (2016). Satisfacción del usuario y calidad del servicio de alojamientos turísticos del estado de Mérida, Venezuela. Revista de Ciencias Sociales, 7(2), 111-131. Recuperado de https://www.redalyc. org/jatsRepo/280/28049145009/html/index.html

Nolazco, J. L., y Bravo-Ortega, C. (2015). Instituciones, recursos naturales y sus impactos en el crecimiento económico: un sistema de ecuaciones simultáneas en panel de datos. MPRA Paper, 74421, 1-49. Recuperado de https:// mpra.ub.uni-muenchen.de/74421/

Organización Mundial de Turismo. (2008). Recomendaciones internacionales para estadísticas de turismo. Recuperado de https://webunwto.s3-euwest-1.amazonaws.com/2019-08/glossary_ES.pdf

Parasuraman, A., Zeithaml, V., y Berry, L. (1985). A conceptual model of service quality and its implications for future research. Journal of Marketing, 49(4), 41-50. doi: 10.1177/002224298504900403

PEMEX. (2011). Anuario Estadístico de PEMEX.

PEMEX. (2016). Anuario Estadístico de PEMEX.

PEMEX. (2018). Anuario estadístico de PEMEX.

Pérez, A., Rivas, M., Martínez, D., y Venegas, J. A. (2018). Reestructuración de PEMEX y su efecto en los salarios y el mercado de trabajo en Ciudad del Carmen, Campeche. Análisis Económico, 82(33), 111-124. doi: 10.24275/uam/ azc/dcsh/ae/2018v33n82/Perez

Pérez, C. (2004). Técnicas de análisis multivariante de datos. Aplicaciones con SPSS. Madrid: Pearson Educación, S. A.

Portal, B. F., Espinoza, J. C., y Carreño, M. (2014). Impacto de la demanda de un turismo social en la sustentabilidad de la actividad en el balneario de El Quisco, Provincia de San Antonio-Chile. Investigaciones Científicas, Boletín del Instituto de Geografía, (83), 102-115. doi: 10.14350/rig.34429

Ramírez, J., García, T., y Cano, M. (2013). Diagnóstico de factores de competitividad de una empresa hotelera. Ciencia Administrativa, 2, 9-18. Recuperado de https: / / www.redalyc.org/jatsRepo/880/88055200021/88055200021.pdf

Ramsaran-Fowdar, R. R. (2007). Developing a service quality questionnaire for the hotel industry in Mauritius. Journal of Vacation Marketing, 13(1), 19-27. doi: 10.1177/1356766706071203

Ríos, M. A., Ortega, F. J., y Matilla, M. (2016). La estancia perfecta en hoteles de 4 y 5 estrellas de Sevilla a través del análisis de los comentarios en TripAdvisor -Determinación de los principales ítems. International Journal of Information System and Tourism, 1(1), 8-25. Recuperado de http://www. uajournals.com/ojs/index.php/ijist/article/view/168/145 
Robledo-Galván, H., Meljem-Moctezuma, J., Fajardo-Dolci, G. y Olvera-López, D. (2012). De la idea al concepto en la calidad en los servicios de salud. Revista CONAMED, 17(4), 172-175. Recuperado de https://dialnet.unirioja.es/ servlet/articulo?codigo $=4175520$

Robles, C., Martínez, E., Alviter, L. E., y Ortega, A. O. (2016). Percepción de la cultura de la calidad en propietarios de microempresas familiares del sector servicios. Acta Universitaria, 26(2), 77-92. doi: 10.15174/au.2015.843

Ruizalba, J. L., Vallespín, M., y Pérez-Aranda, J. (2015). Gestión del conocimiento y orientación al marketing interno en el desarrollo de ventajas competitivas en el sector hotelero. Investigaciones Europeas de Dirección y Economía de la Empresas, 21(2), 84-92. doi: 10.1016/j.iedee.2014.03.001

Salas, C. M., y López, A. (2019). Efectos espaciales de la tematización cultural para la recreación y el turismo en los corredores peatonales del centro histórico de la Ciudad de México. Investigaciones Geográficas, 98(1), 1-17. doi: 10.14350 / rig. 59763

Sánchez, L. D., Sánchez, J., y Hernández, S. G. (2010). Estudio de la satisfacción del usuario de hoteles cinco estrellas de la ZMG: una aplicación del instrumento Servqual. Mercados y Negocios: Revista de Investigación y Análisis, (22), 109-128. Recuperado de https://dialnet.unirioja.es/servlet/articulo?codigo $=6149955$

Scheyvens, R., y Russell, M. (2012). Tourism and poverty alleviation in Fiji: comparing the impacts of small and large-scale tourism enterprises. Journal of Sustainable Tourism, 20(3), 417-436. doi: 10.1080/09669582.2011.629049

Sebastián, R., Claudio, A., y Larios, E. (2017). Factores determinantes en la viabilidad del turismo de negocios en Puebla (México) como oportunidad de desarrollo económico. Revista Interamericana de Ambiente y Turismo, 13(2), 184-193. doi: 10.4067/S0718-235X2017000200184

Secretaría de Turismo (SECTUR). (2018). Compendio estadístico de la actividad hotelera. Recuperado de https://www.datatur.sectur.gob.mx

Šerić, M., y Gil, I. (2011). Análisis de los hoteles de alta categoría de Croacia desde el enfoque hotel-huésped. Investigaciones Europeas de Dirección y Economía de la Empresa, 17(3), 127-149. doi: 10.1016/S1135-2523(12)60124-5

Sierra, B. (1999). Hotelqual: una escala para medir la calidad percibida en servicios de alojamientos. Estudios Turísticos, 139(199), 87-92. Recuperado de https: / / dialnet.unirioja.es/ejemplar/148728

Stefano, N. M., Casarotto, N., Barichelo, R., y Sohn, A. P. (2015). A fuzzy Servqual based method for evaluated of service quality in the hotel industry. Procedia CIRP, 30, 433-438. doi: 10.1016/j.procir.2015.02.140

Ulacia-Oviedo, Z. (2012). Determinación de factores para la medición y evaluación de la calidad del servicio hotelero. Revista de la Escuela Jacobea de Posgrado, 3, 1-32. Recuperado de https://www.jacobea.edu.mx/revista/ numeros/numero3/1.determinacion_factores_medicion_evaluacion_servicio_hotelero.pdf

Vergara, J. C., Quesada, V. M., y Blanco, I. (2011). Análisis de la calidad en el servicio y satisfacción de los usuarios en dos hoteles de cinco estrellas de la ciudad de Cartagena (Colombia) mediante un modelo de ecuaciones 
estructurales. Ingeniare, Revista Chilena de Ingeniería, 19(1), 420-428. doi: 10.4067/S0718-33052011000300011

Vidal, M. (2004). Tierra de Gracia y buenos negocios. Debates IESA, Ed. Aniversario, 11(1), 47-63.

Villena, M. (2014). Turismo de negocios: análisis del gasto diario de los turistas de negocios. Anuario Jóvenes Investigadores, 1(1), 78-80. Recuperado de http://hdl.handle.net/10317/4425

Zeithaml, V., Berry, L., y Parasuraman, A. (1993). The nature and determinants of customer expectations of service. Journal of the Academy of Marketing Science, 21(1), 1-12. doi: 10.1177/0092070393211001 